\title{
Anti- Müllarian hormone (AMH) as an indicator of ovarian reserve in acne patients treated with isotretinoin
}

M.A.Ibrahim ${ }^{1}$, G.M.Abdel-Khalik ${ }^{1}$, A.S.Soliman ${ }^{2}$, and AM.Haroun ${ }^{1}$

${ }^{1}$ Dermatology and Andrology, Dept., Faculty of Medicine, Benha Univ., Benha, Egypt

${ }^{2}$ Gynecology and Obstetrics, Dept., Faculty of Medicine, Benha Univ., Benha, Egypt

Email: dermagenius@gmail.com

\begin{abstract}
Background: Isotretinoin suppresses sebum development by inducing sebocyte apoptosis, which is induced by the expression of tumour necrosis factor-related apoptosis-inducing ligand, insulin-like growth factor binding protein-3, and neutrophil gelatinase-associated lipocalin, which is induced by isotretinoin. While apoptosis could play a role in the drug's key mechanism of action, it may also be to blame for the majority of isotretinoin's side effects, including ovarian reserve impairment. The aim of this study was to see if AMH could be used as a measure of ovarian reserve in isotretinoin-treated acne patients. The participants in this study were 66 female patients in their reproductive years who had various degrees of acne. All of the patients were chosen from the Dermatology and Andrology Department of Benha University Hospitals' outpatient clinic. The research lasted anywhere from 6 to 12 months. The average age of the patients in this study was 25 years, with a standard deviation of 6 years. There was no substantial difference in AMH before and after Isotretinoin therapy. The percentage change in AMH after treatment had no important relationship with the patients' age, Isotretinoin dosage, or treatment period. Isotretinoin has a negative impact on the ovaries, according to the findings. More research is required to gain a better understanding of this subject and to see if the effects are permanent.
\end{abstract}

Keywords: Anti-Müllarian, AMH, ovarian, acne, isotretinoin.

\section{Introduction}

Acne vulgaris is a long-term skin disease affecting the pilosebaceous unit, with superficial, deep, and secondary lesions. Superficial lesions include comedones (black heads and white heads), papules, and pustules; deep lesions include noduler and pseudocyst; and secondary lesions include excoriations, erythematous macules, pigmented macules, and scars. Acne primarily affects skin with a high number of sebaceous glands, such as the face, upper chest, and back [1].

Many etiological factors contribute to the development of acne, including genetics, which is thought to be the cause of $80 \%$ of cases, as well as stress, diet, and lifestyle infection by Propionebacterium acne. Acne is also an androgen-dependent disease with a weakened adaptive immune system [2].

Despite the fact that most patients have natural hormone levels, it has been proposed that androgens play a role in acne pathogenesis. Acne is caused by localised (cutaneous) androgen overproduction or high expression and responsiveness of androgen receptors. Oral isotretinoin, a common and effective medication that has been used for many years, is the treatment of choice for acne vulgaris. It prevents bacterial replication and decreases sebum secretion, resulting in acne shrinkage. Isotretinoin was first approved by the Food and Drug Administration (FDA) in May 1982 for serious, recalcitrant, and nodular acne. Since then, it has been used for a variety of other conditions, including treatment-resistant acne, scarring acne, and acne that causes significant psychological distress [3].

All-trans retinoic acid (RA), on the other hand, is used in the initial treatment of acute promyelocytic leukaemia and plays a key role in the maturation of malignant promyelocytes into mature neutrophils [4].

Ovarian reserve refers to the amount of goodquality oocytes left in a woman's ovaries when she gets older. This is attributable to the apoptotic loss of primordial follicles. Common ovarian reserve markers include early follicular phase FSH, estradiol, and inhibin $B$. The most sensitive noninvasive markers of ovarian reserve tend to be ovarian antral follicle count (AFC) and plasma anti-Müllerian hormone (AMH) levels [5].

$\mathrm{AMH}$ is a valid ovarian reserve marker. AMH concentrations in the blood have been linked to a reduction in response capacity, and its expression is lost when a follicle becomes atretic. AMH has been proposed as a possible biomarker for ovarian reserve following chemotherapy and exposure to other gonadotoxic agents in recent studies [6].

AMH is an earlier indicator of ovarian reserve in the ageing phase than other known ovarian hormones including FSH, inhibin B, or oestradiol, and has been shown to have stable serum levels during the menstrual cycle [7].

The aim of this research is to see whether AMH can be used as a measure of ovarian reserve in acne patients taking isotretinoin.

\section{Patients and methods}

This study is cohort prospective observational study. And included 66 female patients of reproductive age suffering from different degrees of acne. All patients was selected from the outpatient clinic of Dermatology and Andrology Department of Benha University Hospitals.

An informed consent was obtained from all participants. The study was approved by the local ethics committee on research Involving human subjects of Benha Faculty of Medicine.

\subsection{Inclusion criteria}

- Patients with acne vulgaris, who don't receive systemic treatment in the last 4 weeks.

- Females with regular menstrual cycles and normal ovarian morphologies, confirmed by ultrasonography. 


\subsection{Exclusion criteria}

- Any dermatologic disorder other than acne.

- Any systemic disease(hypertension, thromboembolic disease, diabetes mellitus, cardiovascular events, Cushing's disease, positive malignancy, congenital adrenal hyperplasia, liver disease, psychotic disorders, thyroid disorders).

- pregnancy or lactation

- All patients were subjected to history taking, clinical examination and lab. investigations, Serum AMH concentrations were determined using an enzymelinked immunosorbent assay kit before isotretinoin treatment and then it was measured six months after last dose isotretinoin treatment.

\subsection{Statistical analysis}

Data entry, processing and statistical analysis was carried out using Statistical package for social sciences (IBM-SPSS), version 24 (May 2016); IBM- Chicago,
USA will be used for statistical data analysis. Tests of significance (Kruskal-Wallis, Wilcoxon's, Chi square, logistic regression analysis, and Spearman's correlation) were used. Data were presented and suitable analysis was done according to the type of data (parametric and nonparametric) obtained for each variable. P-values less than $0.05(5 \%)$ was considered to be statistically significant.

\section{Results}

This study included sixty-six female patients of reproductive age suffering from different degrees of acne. All patients were selected from the outpatient clinic of Dermatology and Andrology Department of Benha University Hospitals. The mean age of the studied patients was 25 years, with a standard deviation of 6 years. ALL patients (100.0\%) showed gradual onset. Regarding course, 95\% showed progressive course, and only $5.0 \%$ showed stationary course Table (1)

Table (1) General characteristics of the studied patients.

\begin{tabular}{lccc}
\hline \multicolumn{3}{c}{ General characteristics } \\
\hline Age (years) & Mean \pm SD & & $25 \pm 6$ \\
Onset & Gradual & n (\%) & $20(100.0)$ \\
Course & Progressive & n (\%) & $19(95.0)$ \\
& Stationary & n (\%) & $1(5.0)$ \\
\hline
\end{tabular}

Regarding Isotretinoin dose, the mean dose was 0.6 with a standard deviation of 0.2 . The mean duration of treatment was five months with a standard deviation of 1 month.

Table (2) Correlation between AMH percent change after treatment and other parameters.

\begin{tabular}{lcc}
\hline & \% change of AMH after treatment \\
\hline & r & P-value \\
Age (years) & -0.026 & 0.912 \\
Dose & 0.347 & 0.134 \\
Duration (months) & -0.319 & 0.170 \\
\hline
\end{tabular}

Spearman's correlation was used

\section{Discussion}

Isotretinoin is the only acne treatment that affects all of the main etiological variables. It achieves this remarkable efficacy by interfering with cell division, differentiation, survival, and apoptosis. It has antiinflammatory properties, reduces sebum content, influences comedogenics, lowers surface and ductal P. acnes, and influences comedogenics. Within 6 weeks, a dosage of $0.5-1.0 \mathrm{mg} / \mathrm{kg} /$ day significantly decreases sebum excretion by the order of $90 \%$. Isotretinoin, unlike tretinoin (all-trans retinoic acid), has little or no ability to bind to cellular retinol-binding proteins or retinoic acid nuclear receptors (RARs and RXRs), but it can act as a pro-drug that is converted intracellularly to metabolites that are nuclear receptor agonists [8].

The current study found that the average dose of isotretinoin was 0.6 , with a standard deviation of 0.2 . The average treatment time was five months, with a onemonth standard deviation. $r=$ Correlation coefficient

The amount of high quality oocytes left in the ovaries is referred to as ovarian reserve. The loss of primordial follicles due to apoptosis reduces a woman's ovarian reserve as she gets older. Common ovarian reserve markers include early follicular phase $\mathrm{FSH}$, oestradiol, and inhibin B. The most sensitive noninvasive markers of ovarian reserve tend to be the ovarian antral follicle count (AFC) and plasma anti-Mullerian hormone $(\mathrm{AMH})$ levels. $\mathrm{AMH}$ is a valid ovarian reserve marker. AMH levels in the blood have been linked to a reduction in response potential, and its expression disappears when a follicle becomes atretic [9].

AMH has been proposed as a possible biomarker for ovarian reserve following chemotherapy and other gonadotoxic agents in recent studies. AMH is an earlier indicator of ovarian reserve throughout the ageing phase than other known ovarian hormones including FSH, inhibin B, or oestradiol, and has been shown to have stable serum levels during the menstrual cycle. 
Isotretinoin has been shown to have a variety of side effects. Isotretinoin has been shown to be harmful to rat ovaries in a recent study [10].

AMH did not demonstrate any major differences between pre and post-Isotretinoin treatment in the sample we had. The p-value for this study was 0.126 . Patients' age $(\mathrm{r}=-0.026 \& \mathrm{P}$ value $=0.912)$, Isotretinoin dosage $(\mathrm{r}$ $=0.347 \& \mathrm{P}$ value $=0.134)$, and care time $(r=-0.319 \&$ $\mathrm{P}$ value $=0.17)$ had no substantial association with percent improvement in $\mathrm{AMH}$ after treatment.

Can et al., [11], found that the mean AMH level of the treatment group was significantly lower at month 6 than the level prior to treatment and that of the control group ( $\mathrm{p} 0.001, \mathrm{p}=0.013)$, despite no substantial difference between the treatment group and the control group prior to treatment $(\mathrm{p}=0.967)$. At month 6 , mean AMH levels in both the low-dose and high-dose groups were significantly lower than before treatment $(\mathrm{p}=0.005$, p0.001). When the 6th month AMH levels of the low and high-dose treatment groups were compared to controls, the high dose group's mean AMH level was lower, whereas the low-dose treatment group's mean AMH level was not significantly different from the control group $(\mathrm{p}=0.009, \mathrm{p}=0,097)$.

According to Aksoy et al., [12], major variations in AMH $[2.20 \mathrm{ng} / \mathrm{ml}$ (25th-75th percentile 1.14-4.07) vs. $1.31 \mathrm{ng} / \mathrm{ml}(0.32-2.28) ; \mathrm{p} \mathrm{0.001]}$, total AFC [16 (14$18.25)$ vs. 12.5 (10-15); p 0.001], and total OV (ovarian volume) [23 $\mathrm{ml}(18-29) \mathrm{v}$

Korkmaz et al. [13] measured serum AMH levels before administering isotretinoin to all rats in their sample. The lack of a statistically significant difference between the study groups in terms of pre-isotretinoin AMH values resulted in two identical study groups with comparable ovarian reserves, except variations related to intrinsic rat ovarian function. Then, according to previous data [10], we compared the results of 7.5 $\mathrm{mg} / \mathrm{kg} /$ day isotretinoin treatment to no treatment.

In contrast to previous research, Korkmaz et al. [13] tested AMH values prior to drug administration, as well as $\mathrm{AMH}$ values immediately after and one month after the last dose of isotretinoin administration, to see whether there was any drug impact and how long it lasted. While there were no major differences between the groups, the isotretinoin treatment group's AMH values were slightly lower on the day immediately following the last dose $(\mathrm{p}=0.02)$ than the pretreatment values. As compared to the values obtained immediately after the last dose of isotretinoin, the mean AMH values increased on the day 1 month after the last dose, but the difference was not important. Furthermore, no statistically significant changes in AMH values 1 month after the last dose of isotretinoin relative to pretreatment values were found. These results indicate that after isotretinoin cessation, AMH concentrations increased, providing additional evidence for the first time in the literature, suggesting that the impact of isotretinoin on ovarian reserves could be reversible [13].

The toxic effects of isotretinoin on the ovaries have recently been highlighted by the discovery of lower levels of $\mathrm{AMH}$ in rats and women exposed to the compound. The granulosa cells of the preantral and small antral follicles of the ovary contain $\mathrm{AMH}$, a dimeric glycoprotein. Although there are no reliable tools to test for early ovarian ageing, $\mathrm{AMH}$ is known to be a good biomarker for predicting ovarian reserve by providing a way to investigate the non-growing follicle pool and the ability to assess early ovarian ageing, especially in the $10 \%$ of women in the general population who may experience 'early ovarian ageing' before the age of 40 [14].

In a rat model, Abali et al. [10] discovered that after 7.5 and $15 \mathrm{mg} / \mathrm{kg} /$ day isotretinoin treatment, serum $\mathrm{AMH}$ concentrations decreased and the percentage of atretic follicles increased, indicating that isotretinoin has a toxic effect on the ability of ovarian reserve. Despite the importance of the data collected, there was insufficient information to assess if the devastating impact is permanent. As a result, the authors were advised to conduct future experiments that would demonstrate the length of the toxic impact.

Furthermore, Cinar et al., [15] found that all OR (ovarian reserve) parameters were evaluated, and mean $\mathrm{AMH}, \mathrm{OV}$, and TAFC (total antral follicle count) values at the start and 12 months after treatment cessation were statistically similar, despite the fact that these three parameters decreased significantly just after the treatment.

Isotretinoin therapy also reduced Ferriman-Gallwey score, free testosterone, insulin level, haemoglobin level, acne score, and ovarian volume, according to Acmaz et al., [16].

\section{Conclusion}

Isotretinoin is a commonly used acne medication with well-documented side effects. Fertility is a significant problem for young adult patients and their parents. Despite the fact that the decrease in AMH levels is substantial, this impact may be reversed. Studies examining $\mathrm{AMH}$ levels after a reasonable amount of time has passed after treatment termination are needed.

\section{References}

[1] S.Knutsen-Larson, A.L.Dawson, C.A.Dunnick, and R.P.Dellavalle, "Acne vulgaris: pathogenesis, treatment, and needs assessment," Dermatol. Clin., vol. 3, pp. 99-106, 2012.

[2] K.Bhate and H. C.Williams, "Epidemiology of acne vulgaris," Br. J. Dermatol., vol. 168, pp. 474-485, 2013.

[3] A.J.Cooper and A.R.A Board, "Treatment of acne with isotretinoin: recommendations based on Australian experience," Australas. J. Dermatol., vol. 44, pp. 97-105, 2003.

[4] M.S.Tallman and J.K.Altman, "How I treat acute promyelocytic leukemia," Blood, vol. 114, pp. 5126-5135, 2009.

[5] K.P.Tremellen, M.Kolo, A.Gilmore, and D. N. Lekamge, "Anti-müllerian hormone as a marker 
of ovarian reserve," Aust. New Zeal. J. Obstet. Gynaecol., vol. 45, pp. 20-24, 2005.

[6] R.A.Anderson and D.A.Cameron, "Pretreatment serum anti-müllerian hormone predicts long-term ovarian function and bone mass after chemotherapy for early breast cancer," J. Clin. Endocrinol. Metab., vol. 96, pp. 1336-1343, 2011.

[7] R.A.Anderson, "What does anti-M üllerian hormone tell you about ovarian function?," Clin. Endocrinol. (Oxf)., vol. 77, pp. 652-655, 2012.

[8] A.Layton, "The use of isotretinoin in acne," Dermatoendocrinol., vol. 1, pp. 162-169, 2009.

[9] R.Fleming .Can anti-Müllerian hormone concentrations be used to determine gonadotrophin dose and treatment protocol for ovarian stimulation?," Reprod. Biomed. Online, vol. 26, pp. 431-439, 2013.

[10]R.Abali ."Decreased ovarian reserve in female Sprague-Dawley rats induced by isotretinoin (retinoic acid) exposure," Reprod. Biomed. Online, vol. 27, pp. 184-191, 2013.

[11]P.K.Can, E.Kocatürk, V.Mihmanl1, V.Sucu, E.N.Değirmentepe, and U. Kızıltaç, "The evaluation of ovarian reserve and menstrual irregularities in female patients treated with systemic isotretinoin.," Turkish Arch. Dermatology Venerol., vol. 54, pp. 43-56, 2020.

[12]H. Aksoy ."The effect of isotretinoin on ovarian reserve based on hormonal parameters, ovarian volume, and antral follicle count in women with acne," Gynecol. Obstet. Invest., vol. 79, pp. 7882, 2015.

[13]E. Korkmaz . "The possibly reversible isotretinoin effect of decreased ovarian reserve in spraguedawley albinos: Part I, biochemical analyses," Gynecol. Obstet. Invest., vol. 82, pp. 72-77, 2017.

[14]P.-Y. Lin ."Evaluation of serum anti-Mullerian hormone as a biomarker of early ovarian aging in young women undergoing IVF/ICSI cycle," Int. J. Clin. Exp. Pathol., vol. 7, pp. 6245, 2014.

[15] S.L.Cinar ."Long-term effect of systemic isotretinoin on female fertility," Cutan. Ocul. Toxicol., vol. 36, pp. 132-134, 2017.

[16] G.Acmaz . "The effects of oral isotretinoin in women with acne and polycystic ovary syndrome," Biomed Res. Int., vol. 2019, pp, 3442, 2019. 